\title{
Role of the ubiquitin-like protein Hub1 in splice-site usage and alternative splicing
}

\author{
Shravan Kumar Mishra ${ }^{1}$, Tim Ammon ${ }^{1}$, Grzegorz M. Popowicz ${ }^{2}$, Marcin Krajewski ${ }^{2, \dagger}$, \\ Roland J. Nagel ${ }^{3, \dagger}$, Manuel Ares Jr ${ }^{3}$, Tad A. Holak ${ }^{2}$, and Stefan Jentsch ${ }^{1}$ \\ ${ }^{1}$ Department of Molecular Cell Biology, Max Planck Institute of Biochemistry, Am Klopferspitz 18, \\ 82152 Martinsried, Germany. \\ ${ }^{2}$ NMR Spectroscopy, Max Planck Institute of Biochemistry, Am Klopferspitz 18, 82152 \\ Martinsried, Germany. \\ ${ }^{3}$ Center for Molecular Biology of RNA, Department of Molecular, Cell \& Developmental Biology, \\ University of California, Santa Cruz, California 95064, USA.
}

\begin{abstract}
Alternative splicing of pre-messenger RNAs diversifies gene products in eukaryotes and is guided by factors that enable spliceosomes to recognize particular splice sites. Here we report that alternative splicing of Saccharomyces cerevisiae SRC1 pre-mRNA is promoted by the conserved ubiquitin-like protein Hub1. Structural and biochemical data show that Hub1 binds non-covalently to a conserved element termed HIND, which is present in the spliceosomal protein Snu66 in yeast and mammals, and Prp38 in plants. Hub1 binding mildly alters spliceosomal protein interactions and barely affects general splicing in $S$. cerevisiae. However, spliceosomes that lack Hub1, or are defective in Hub1-HIND interaction, cannot use certain non-canonical $5^{\prime}$ splice sites and are defective in alternative $S R C 1$ splicing. Hub1 confers alternative splicing not only when bound to HIND, but also when experimentally fused to Snu66, Prp38, or even the core splicing factor Prp8. Our study indicates a novel mechanism for splice site utilization that is guided by non-covalent modification of the spliceosome by an unconventional ubiquitin-like modifier.
\end{abstract}

Covalent modification of proteins by ubiquitin and related proteins (collectively called ubiquitin-like modifiers, UBLs) often critically alters substrate activity by influencing metabolic stability, binding behaviour or localization ${ }^{1}$. The switch-like properties of UBLs are crucial for pathways that regulate, for example, signal transduction, protein sorting, DNA repair, and development ${ }^{1}$. Covalent conjugation of a UBL to a substrate's target

(C)2011 Macmillan Publishers Limited. All rights reserved

Correspondence and requests for materials should be addressed to S.J. (Jentsch@biochem.mpg.de). .

†Present addresses: Institut für Biomedizinische Technik, ETH Zürich, Wolfgang-Pauli-Str. 10, 8093 Zürich, Switzerland (M.K.); Enzymology Research and Development, Life Technologies, 850 Lincoln Centre Drive, Foster City, California 94404, USA (R.J.N.).

Full Methods and any associated references are available in the online version of the paper at www.nature.com/nature.

Supplementary Information is linked to the online version of the paper at www.nature.com/nature.

Author Contributions S.K.M. (S. cerevisiae, S. pombe), T.A. (mammalian) and S.J. designed, obtained and analysed the genetic, biochemical and functional data; G.M.P, M.K. and T.A.H. the structural data; R.J.N. and M.A. Jr the splicing array data. S.J. and S.K.M. wrote the paper, and all authors contributed to the manuscript.

Author Information Coordinates and the experimental structural factors of both complexes have been deposited in the PDB under the following codes: Hub1-HIND-I, 3PLU; Hub1-HIND-II, 3PLV. Reprints and permissions information is available at www.nature.com/reprints.

The authors declare no competing financial interests.

Readers are welcome to comment on the online version of this article at www.nature.com/nature. 
residue is ATP dependent, involves an enzyme cascade, and usually requires a free diglycine (GG) motif at the protruding carboxy-terminal end of the UBL. Archetypal UBLs (ubiquitin, SUMO, Rub1 (also known as Nedd8)) are expressed as inactive precursors with $\mathrm{C}$-terminal extensions. These extensions are removed by UBL-specific proteases, exposing the crucial C-terminal GG motif. Enzymes of this class also mediate UBL deconjugation, thus making the UBL-dependent switch reversible ${ }^{1}$.

Hub1 (homologous to ubiquitin; known as UBL5 or beacon in mammals), another evolutionarily highly conserved UBL, is unique in lacking a protruding C-terminal tail with a GG motif. Instead, Hub1 possesses a C-terminal double tyrosine (YY) motif, followed by a non-conserved amino acid residue ${ }^{2,3}$. Although Hub1 from various organisms has been studied to some extent ${ }^{4-8}$, its function remains poorly understood. Whereas $S$. cerevisiae cells deficient in Hub1 are viable and exhibit only minor phenotypes under normal growth conditions ${ }^{6,7}$, the corresponding mutant of Schizosaccharomyces pombe is lethal ${ }^{4,8}$. One study reported that Hub1 forms covalent conjugates similar to ubiquitin and proposed that Hub1 is synthesized as a precursor and matured by processing C terminally of the YY motif $^{6}$. However, no Hub1-specific processing, conjugation or deconjugation enzymes have been identified. Further studies have ruled out that Hub1 functions as a covalent protein modifier ${ }^{7,8}$; in fact, Hub1 was found to bind proteins non-covalently and independently of ATP, and the YY motif was shown to be nonessential ${ }^{7,8}$.

Hub1 has been linked to various physiological functions, including cell cycle progression and polarized growth ${ }^{6}$, the mitochondrial unfolded protein response ${ }^{9}$, and mRNA splicing ${ }^{4,8}$. Conditional mutants of $S$. pombe HUB1 show moderate RNA splicing defects, particularly at high temperatures, and Hub1 formed a non-covalent association with the spliceosomal (U4/U6.U5) tri-small nuclear ribonucleoprotein particle (snRNP) protein Snu66 (refs 4, 8). However, how the Hub1-Snu66 interaction affects splicing is unclear. It has been proposed that Hub1 is required for the nuclear localization of Snu66 (ref. 4), but Hub1 may affect the spliceosome directly and influence its activity.

Here we show that Hub1, through binding to Snu66, modifies the spliceosome in a way that enables it to tolerate and use certain non-canonical $5^{\prime}$ splice sites. We discovered that Hub1 binds Snu66 through an element called HIND in a unique, sequence-specific manner. We propose that Hub1 operationally resembles UBLs, with the important difference that Hub1 modifies substrates through non-covalent binding.

\section{Hub1 binds to HINDs of spliceosomal proteins}

Hub1 has been shown to bind the tri-snRNP protein Snu66 in yeast two-hybrid (Y2H) assays ${ }^{4,10}$. To verify this interaction in vivo, we raised antibodies specific for yeast Hub1 and Snu66 for co-immunoprecipitation assays. The antibodies against Hub1 bring down Snu66 from cell extracts and vice versa (Fig. 1a, b). Similar experiments with an altered form of Hub1 (YY changed to AA) showed that binding to Snu66 was independent of the YY motif (data not shown).

Amino-terminal fragments of Snu66 were both sufficient and required for Hub1 binding. Inspection of the protein sequence revealed two highly similar elements (72\% identity) arranged in tandem (Fig. 1c, d). Fragments harbouring these repeats, either singly or in tandem, strongly interacted with Hub1 in Y2H assays (Supplementary Fig. 1a). Notably, the elements do not seem to bind ubiquitin or SUMO in Y2H assays, and Hub1 does not seem to bind classical ubiquitin-binding motifs like UBA or UIM (data not shown). The minimal polypeptide sequence defined by this assay was 18-19 amino acids long; because it had no obvious similarity to known motifs, we termed it HIND (Hub1-interaction domain). GST pull-down assays with purified proteins confirmed that Hub1-Snu66 binding is direct and 
mediated via HIND (Supplementary Fig. 1b, c). Gel filtration analysis indicates that the Nterminal domain of Snu66 can bind two Hub1 molecules (Supplementary Fig. 1d).

Snu66 proteins (also known as SART1) from Saccharomycotina (for example, S. cerevisiae, Candida) and some Stramenopiles possess two N-terminal HINDs, but the homologues from $S$. pombe and vertebrates possess only one HIND at this position (Fig. 1d). As Hub1 binds Snu66 via its HIND in $S$. pombe and in humans also (Supplementary Fig. 2a-e), the mechanism of Hub1 recruitment seems to be conserved. Intriguingly, plant Snu66 homologues (and also for example, Amoebazoa) lack HIND sequences; this absence is compensated by HINDs found in C-terminal extra domains of proteins related to the spliceosomal protein Prp38 (Fig. 1d and Supplementary Fig. 2f). Furthermore, in Plasmodium, functional HINDs are present in both Snu66 and Prp38 homologues, as they bind Hub1 and involve the same surface interface (Supplementary Fig. 2g, h). Because in yeast and mammalian cells Snu66 and Prp38 are constituents of the spliceosome ${ }^{11}$, HINDs may be evolutionarily associated (and perhaps restricted) to splicing proteins. Moreover, as the identity of the HIND-bearing spliceosomal protein seems to be flexible, HINDs may function irrespective of their exact positioning within the spliceosome (see later). By extension, this observation indicates that Hub1 may affect spliceosome activity as a whole, rather than functionally modulate the protein it directly binds. As Hub1 colocalizes with Snu66 in nuclear speckles in human cells (Supplementary Fig. 3a), it seems likely that the function of Hub1 in splicing is conserved.

\section{Structure of Hub1 in complex with HIND}

To determine whether Hub1 functions as a non-covalently acting modifier by interacting with HIND, we sought to gain molecular insights into the Hub1-HIND interaction. The characterization of peptides corresponding to the two HINDs of S. cerevisiae Snu66 (HINDI, 18 amino acids; HIND-II, 19 amino acids) by circular dichroism (CD) and NMR revealed that an isolated HIND peptide is apparently helical in solution (Supplementary Fig. 4a, b), an unusual feature for such short peptides.

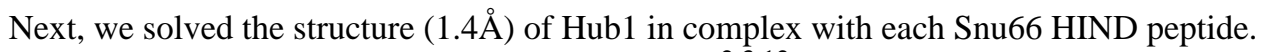
The structure of Hub1 is highly similar to ubiquitin ${ }^{2,3,12}$ (PDB code 1UBI; root mean squared deviation (r.m.s.d.) of $0.88 \AA$ for the main chain heavy atoms) and $\mathrm{SUMO}^{13}$ (PDB code $1 \mathrm{~A} 5 \mathrm{R}$; r.m.s.d. of $1.72 \AA$ ), and consists of a half-open $\beta$ barrel completed with two flanking $a$ helices; the secondary structure elements have a $\beta \beta a \beta a \beta$ pattern (Fig. 1e, Supplementary Figs 5, 6 and Supplementary Table 2).

The observed interactions of Hub1 with the Snu66 HIND-I and HIND-II peptides are almost identical (Fig. 1e and Supplementary Fig. 5a). The bound peptide forms an 11-residue helix (Ile 3-Leu 13, numbering of HIND-I) with its C-terminal part flipped over along the peptide helix (Fig. 1e and Supplementary Fig. 5a). The whole Hub1-HIND interface has a surface of about $500 \AA^{2}$. On HIND binding, the main-chain fold of Hub1 does not change, but a number of side chains are significantly affected (Supplementary Fig. 5b). The Hub1-Snu66 interactions seen in the X-ray structures were fully corroborated by our mutational studies. For example, replacement of the salt-bridge-forming residues Asp 22 of Hub1 and Arg 16(47) (number in parentheses refers to residues in HIND-II) of Snu66 by Ala abolished Hub1-Snu66 binding (Supplementary Fig. 5c-f).

Most unexpectedly, the structure of Hub1-HIND revealed a new binding paradigm unseen in interactions of ubiquitin and ubiquitin-like proteins with their binding partners. Most ubiquitin-binding modules of ubiquitin receptors bind to the hydrophobic surface of ubiquitin centred on Ile 44 (ref. 14), which is almost exactly on the site opposing the HINDbinding face of the ubiquitin fold (Supplementary Fig. 6a). The ubiquitin interactions with 
ubiquitin receptors have mostly hydrophobic character and are usually weak $\left(K_{\mathrm{d}} \geq 100 \mu \mathrm{M}\right)$, whereas the Hub1-HIND interaction comprises a strong salt bridge accompanied by several hydrophobic contacts and high affinity $\left(K_{\mathrm{d}}\right.$ Hub1-Snu66: $0.59 \pm 0.07 \mu \mathrm{M} ; K_{\mathrm{d}}$ Hub1-HINDI: $1.69 \pm 0.27 \mu \mathrm{M}$ ). The mode of interaction of Hub1-HIND is also clearly different from that seen for SUMO interacting with SUMO-interaction motifs (SIM) ${ }^{15}$, although SIMs bind to a similar surface of the ubiquitin fold (Supplementary Fig. 6b).

By performing heteronuclear single quantum coherence (HSQC) NMR, we found that ${ }^{15} \mathrm{~N}^{2} \mathrm{H}$-labelled Snu66, including its HIND-bearing $\mathrm{N}$-terminal domain, is unstructured (Supplementary Fig. 7a, b). As co-expression of Hub1 significantly increased the solubility of Snu66 in bacteria (Supplementary Fig. 7c), we speculated that Hub1 might influence Snu66 folding. Indeed, when unlabelled Hub1 was titrated into ${ }^{15} \mathrm{~N}^{2} \mathrm{H}$-labelled Snu66, several distinct peaks appeared, indicating that a part of the protein acquires structure after Hub1 binding (Supplementary Fig. 7a, b). Comparison of an N-terminal 65-amino-acid fragment and full-length Snu66 revealed that Hub1-induced folding is restricted to the HIND-containing $\mathrm{N}$ terminus of Snu66. This finding further corroborates the idea that Hub1 modifies the spliceosome rather than modulating the properties of an individual binding partner.

\section{Hub1 modifies the spliceosome}

Snu66 is a conserved component of the spliceosomal tri-snRNP complex ${ }^{16,17}$. To investigate whether Hub1 alters the composition of the spliceosome, we immunoprecipitated Snu66 from wild-type, $\triangle h u b 1$ or $H U B 1$-overexpressing strains ( $\triangle$ snu66 strain as control), and identified co-purifying proteins by Orbitrap mass spectrometry (Supplementary Fig. 8a-c). The set of identified proteins in all three samples significantly overlapped with the catalogue of proteins in the yeast spliceosomal complex B (penta-snRNP) ${ }^{18,19}$. Although we did not observe major changes in the protein composition of the three samples, in Hub1-deficient cells we reproducibly found an overrepresentation of certain proteins from U1 and U2 snRNPs, but not of the tri-snRNP (Supplementary Fig. 8a-d); by contrast, no significant spliceosomal alterations were observed in the strain overexpressing Hub1. We confirmed these findings by Snu66-directed immunoprecipitation using strains that express tagged versions of selected proteins from the tri-, U1-, and U2-snRNPs from their genomic loci (Supplementary Fig. 8d). We conclude that modification of the spliceosome by Hub1 only moderately affects spliceosome composition, so that the basic makeup of the snRNPs is preserved. Hub1 does bind spliceosomes in vivo, as we could co-immunoprecipitate the central spliceosomal protein Prp8 with Hub1 antibodies, in a reaction that is mediated by Snu66 (Supplementary Fig. 8e).

\section{Functional links to the spliceosome}

Hub1 is present in both cytosol and nucleus in yeasts and mammals, whereas Snu66 appears to be mostly nuclear (Supplementary Fig. 3a, b) ${ }^{4,8}$. It was reported that Hub1 is required for the nuclear localization of Snu66 (ref. 4), but we found that Snu66 is nuclear even in $S$ cerevisiae $\Delta$ hub1 cells, and that a Snu66 variant deficient in Hub1 binding is nuclear in $S$. cerevisiae, $S$. pombe and human cells (Supplementary Fig. 3a, b and data not shown). Although in $S$. pombe HUB1 and SNU66 are essential ${ }^{4,8}$, mutants deficient in Hub1-Snu66 interaction are viable (Supplementary Fig. 10a, b). This finding, together with the detection of significant cytosolic pools of Hub1 and the observation that Hub1 is apparently more abundant in cells than Snu66 (data not shown), indicates that the function of the Hub1 modifier may not be restricted to splicing.

We observed synthetic sickness or lethality of $\Delta h u b 1$ and $\Delta s n u 66$ mutants if they were combined with mutant alleles of a number of spliceosomal genes (Supplementary Fig. 9a, 
b). Notably, the phenotype of $4 h u b 1 \operatorname{prp} 8^{*}$ ( $\operatorname{prp} 8^{*}$ refers to $\operatorname{prp} 8(P 1384 L)$ ) could not be rescued by expressing hub1(D22A), which encodes a Hub1 variant defective in Snu66 interaction (Fig. 2a). Similarly, the lethal $4 s n u 66$ prp $8^{*}$ double mutant could not be complemented by expression of Snu66 variants that lack the two HINDs (Fig. 2b). Thus, we conclude that Hub1, Snu66 and the Hub1-HIND interaction are indeed relevant for splicing.

As noted earlier, HIND elements in $S$. cerevisiae are located within the $\mathrm{N}$-terminal domain of Snu66, but in plants, HIND is present as a C-terminal extension of the spliceosomal protein Prp38 (Fig. 1d). To address whether the specific localization of HIND is important, we fused a HIND element to the C terminus of yeast Prp38 (PRP38-HIND) and determined functionality as above. We found that PRP38-HIND could indeed functionally rescue snu66( $\triangle H I N D)$ in the synthetic lethality assay with $\operatorname{prp} 8^{*}$, and that this activity depends on HIND residues crucial for Hub1 binding (Supplementary Fig. 9d). This finding confirms a remarkable plasticity of the Hub1 modifier, in that the exact positioning of HIND (and thereby of Hub1), on the spliceosome is not critical for function.

The $S$. cerevisiae $\triangle$ hub1 $\Delta$ snu66 double-but not the corresponding single-mutant is temperature sensitive (Supplementary Fig. 9b). This non-epistatic behaviour suggests partially separate functions and may again point to roles of Hub1 in addition to splicing. Whereas the removal of the YY motif does not interfere with Hub1 activity ${ }^{4,7,8}$, Hub1 variants possessing certain charged C-terminal extensions failed to support the vital activity of Hub1 in S. pombe (Supplementary Fig. 10c), although the proteins can still bind HIND (Supplementary Fig. 10d). Moreover, also in $S$. cerevisiae, expression of a Hub1 variant with a short extension (hub1-DD) could not suppress the synthetic sickness of $4 h u b 1$ prp $8^{*}$ (Fig. 2a). Thus, in addition to the HIND-binding surface the area neighbouring the $\mathrm{C}$ terminus of Hub1 is functionally important, perhaps for additional physical interactions.

To investigate the splicing competence of Hub1-deficient cells directly, we used a splicingsensitive microarray that can distinguish spliced from unspliced RNAs of almost all introncontaining $S$. cerevisiae genes ${ }^{20,21}$. Onto this array we hybridized RNA samples from wildtype, $\Delta h u b 1, \Delta$ snu66 and $\Delta h u b 1 \Delta$ snu66 cultures (Fig. 2c). We found virtually no splicing defects in the $\triangle h u b 1$ mutant for all transcripts, with the only discernable exception of $R P L 34 B$, the splicing of which was mildly affected. On the other hand, we noticed small splicing defects for $\Delta$ snu66, which were aggravated if the cells were additionally defective in Hub1 ( 4 hub1 $\Delta$ snu66) (Fig. 2c and Supplementary Table 3). To confirm these data, we isolated RNA from these strains and analysed splicing of a selected set of RNAs by quantitative polymerase chain reaction with reverse transcription (RT-qPCR). Again, splicing was virtually normal in $\Delta h u b 1$ cells, and only a small splicing defect was discernable for $R P L 34 B$ (Supplementary Fig. 11a). However, this small defect in RPL34B splicing is rather unlikely to affect the level of this ribosomal protein, as splicing of $R P L 34 A$, the gene product of which is almost identical to that of $R P L 34 B$, is not affected (data not shown). We found that $H U B 1$ transcripts are inducible by cadmium and on oxidative stress (Supplementary Fig. 11b), but we detected no significant $\Delta$ hubl-specific splicing defects under these (or heat shock) conditions (Supplementary Fig. 11c, d). Thus, Hub1 does not seem to significantly affect general splicing, and splicing defects only occur if the spliceosome has additional deficiencies. This indicates that Hub1 might have a silent (and redundant) role in general splicing, or that Hub1 fulfils a specific, splicing-related function.

\section{Splice-site usage and alternative splicing}

Splice sites in $S$. cerevisiae show very little sequence variation, and alterations in the sequence can affect splicing ${ }^{22}$. To address whether Hub1 affects splice-site usage, we used a 
reporter assay based on an intron-containing $R P 51^{*}-\operatorname{Lac}_{\text {fusion }}{ }^{23}$ (Fig. 3a) monitored by both RT-PCR and $\beta$-galactosidase (LacZ) activity. For the consensus $5^{\prime}$ splice site (GUAUGU), again we observed no splicing defects for $\Delta h u b 1$, but defects for $\Delta s n u 66$, prp $8^{*}$ and $\Delta h u b 1$ prp $8^{*}$ (Fig. 3b). Intriguingly, when we altered the $5^{\prime}$-splice-site sequence in the reporter construct, some $5^{\prime}$-splice-site variants were similarly used in both wild-type and $\Delta h u b 1$ cells, but certain others required specifically the presence of Hub1 for proper splicing (Fig. 3a, b and data not shown). In fact, splicing via the mutant $5^{\prime}$ splice site GUAUAU was nearly as defective in $\triangle h u b 1$ as in $p r p 8^{*}$, and was almost abolished in $\triangle$ hub1 prp8* (Fig. 3b). Importantly, this splicing defect also occurred in strains that only express a Hub1 defective in Snu66 binding (hub1(D22A)), or Hub1 harbouring an abnormal $\mathrm{C}$ terminus (hub1-DD) (Fig. 3c). To corroborate and extend these findings we also used another splicing sensitive $A C T 1-C U P 1$ reporter assay ${ }^{24}$. Again we found splicing defects of $\triangle$ hub1 cells for certain $5^{\prime}$-splice-site alterations (GUAUAUU, GUCUGU), but not significantly for variants of the $3^{\prime}$ splice site ( $\underline{G} / \mathrm{AG}, \underline{\mathrm{U}} / \underline{\mathrm{U}}$ instead of U/AG; slash symbolizes the intron/exon boundary) or the branch point (UECUAAC, UACUACEC instead of UACUAAC) (Supplementary Fig. 12).

Most metazoans exhibit a high variation of $5^{\prime}$ splice sites, and this plasticity is exploited for alternative splicing 22,25 . Regulation of these events seems to be guided by factors that enable the spliceosome to recognize divergent $5^{\prime}$ splice sites. In $S$. cerevisiae, in which $5^{\prime}$ splice sites are nearly invariant, alternative splicing is extremely rare ${ }^{22}$. In this organism, $S R C 1$ is the only known case of alternative splicing leading to two different proteins with different functions ${ }^{26-28}$. In both cases, a single (130- or 126-nucleotide long) intron is excised, yet by using two different, overlapping $5^{\prime}$ splice sites. Notably, both $5^{\prime}$ splice sites

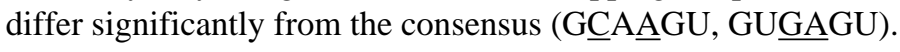

$S R C 1$ was present on the splicing array we used, but the two splice forms could not be individually identified because of their overlapping $5^{\prime}$ splice sites. When we sequenced the complementary DNA across the exon/exon boundaries of spliced $S R C 1$, however, we observed a sequence mixture corresponding to the two splice forms in wild-type cells (and also in $\Delta s n u 66$ and prp $8^{*}$ mutants), but largely only one form (corresponding to splicing via the downstream $5^{\prime}$ splice site) in $\Delta h u b 1$ mutants (Fig. 4a and Supplementary Fig. 13a, b). To corroborate this finding, we made use of a chromosomally expressed TAP-tagged Src1 variant monitoring the expression of the two splice variants ${ }^{28}$. Indeed, splicing via the

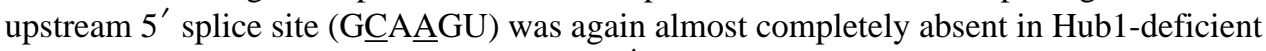
cells, whereas splicing via the downstream $5^{\prime}$ splice site was normal or even more pronounced (Fig. 4b). Moreover, splicing via the upstream $5^{\prime}$ splice site was equally defective in cells that express Hub1 defective in HIND interaction $(h u b 1(D 22 A))$, whereas both forms were reduced in $\Delta s n u 66$ (Fig. 4b). We also detected both forms of the Src1 protein in $\triangle$ prp 17 and prp $8^{*}$ mutants (Supplementary Fig. 13c), indicating that the choice of $S R C 15^{\prime}$ splice site is not significantly altered in these general splicing mutants. Notably, we could make $S R C 1$ splicing via the upstream $5^{\prime}$ splice site independent of Hub1 by mutating this $5^{\prime}$ splice site, but this caused a repression of splicing via the downstream $5^{\prime}$ splice site (Supplementary Fig. 13d). Conversely, certain mutations in the downstream $5^{\prime}$ splice site strongly affected splicing via the upstream $5^{\prime}$ splice site even when Hub1 was present (wildtype cells). We also tested the two $S R C 15^{\prime}$ splice sites individually in the $R P 51^{*}-L a c Z$ reporter and found that in isolation, both non-canonical elements require Hub1 for full splicing activity (Fig. 3a; data not shown). This indicates that the characteristic differential Hub1 dependence of $S R C 1$ alternative splicing requires the tandem arrangement of overlapping $5^{\prime}$ splice sites. In this context it is interesting to note that alternative $S R C 1$ splicing might be regulated, as we found that specifically the Hub1-dependent splicing product is enriched in the G2 phase of the cell cycle (Supplementary Fig. 13e). 
Compared to $S$. cerevisiae, $S$. pombe possesses a much higher number of intron-containing genes, and its splicing machinery resembles more closely its mammalian counterpart ${ }^{22,29}$. Similarly, the sequences of $S$. pombe $5^{\prime}$ splice sites are highly divergent and several cases of alternative splicing are known ${ }^{22,30}$. To address whether Hub1 also mediates selective splicing in $S$. pombe we focused on two different pre-mRNAs. We tested $C D C 2$ (encoding cyclin-dependent kinase), as it possesses four introns with different $5^{\prime}$ splice sites. We found that splicing of intron 3 in particular, which possesses a rarely used $5^{\prime}$ splice site

(GUAUAU; Hub1-dependent in S. cerevisiae; Fig. 3a), needs Hub1 for normal splicing (Supplementary Fig. 14a-d). ZAS1 (encoding a transcription factor), on the other hand, is known to be alternatively spliced as sometimes intron 2 is retained, yielding a larger open reading frame ${ }^{30}$. Again we found that Hub1 is needed for normal $Z A S 1$ splicing and that intron inclusion occurs in hub1 mutants at a much higher frequency (Supplementary Fig. 14d). Thus, Hub1-guided selective splicing is a conserved mechanism, and may occur in all eukaryotes.

Using the $S R C 1$ splicing assay, we examined the significance of our initial observation that HINDs are present on Snu66, Prp38 or both spliceosomal proteins in different organisms. Remarkably, alternative $S R C 1$ splicingwas normalin $\triangle$ hub1 S.cerevisiae cells in which linear (C-terminal) fusions of either Snu66 or Prp38 with Hub1 were chromosomally expressed (Fig. 4c). SRC1 splicing was defective when the Hub1 moiety of the fusions was C-terminally altered (Hub1-DD), but fully functional when the Hub1 moiety was deficient in Snu66 interaction. Hub1 fused to Snu66 $\Delta$ N (lacking the HINDs) via a polyglycine linker may also support Hub1-dependent alternative $S R C 1$ splicing even if free Hub1 and wildtype Snu66 are absent (Fig. 4d). Surprisingly, when we fused HIND-binding defective Hub1 (Hub1(D22A)) to the core spliceosomal protein Prp8, alternative SRC1 splicing was supported (Fig. 4c). These findings indicate that linear Hub1 fusions functionally mimic HIND-mediated Hub1 recruitments, and show that Hub1-dependent effects on splicing act independently of a specific localization on the spliceosome.

\section{Conclusions}

Alternative splicing substantially increases the gene product repertoire and is a major source of cell type differentiation 22,25 . Metazoans in particular use this mechanism extensively, and it is estimated that the majority of human pre-mRNAs undergo alternative splicing ${ }^{22}$. Conventional alternative splicing is largely controlled by positively acting SR (Ser-Arg) proteins and negatively acting heterogeneous nuclear ribonucleoprotein particles (hnRNPs).

We have discovered a new principle for splice-site utilization, which involves non-covalent binding of the ubiquitin-related protein Hub1 to the spliceosome. Notably, Hub1 in $S$. cerevisiae is not required for general splicing and the usage of canonical $5^{\prime}$ splice sites (Fig. $2 \mathrm{c}$ ), but is required for the usage of certain non-canonical $5^{\prime}$ splice sites. Hub1 might function as a 'plasticity factor', which, by relaxing the spliceosome's specificity, enables it to act productively on divergent 5 ' splice sites. Given the high conservation of Hub1 and HIND, and the fact that Hub1-dependent splicing operates through evolutionary divergent spliceosomes, it seems highly likely that Hub1-controlled splicing occurs universally in eukaryotes. SR proteins and hnRNPs involved in spliceosome targeting do not seem to exist in $S$. cerevisiae 22,31 , and thus the Hub1-dependent mechanism may be evolutionarily older. It seems plausible that the more elaborate SR/hnRNP-guided mechanism may have coevolved with the rise in gene complexity to generate multiple different gene products from a single gene.

Hub1 is structurally very similar to ubiquitin and equally highly conserved and ancient, yet the two proteins function in completely different ways. Hub1 binds proteins only non- 
covalently, and at least for splicing, Hub1 seems to functionally alter the complex it binds to, rather than modulating its direct binding partner. Because of this distinctive property, it is attractive to speculate that Hub1 might alter the structure of the spliceosome or provide novel binding surfaces for physical interactions.

\section{METHODS}

\section{Yeast strains, plasmids and DNA techniques}

$S$. cerevisiae and $S$. pombe strains are listed in Supplementary Table 1. Yeast growth assays were performed by spotting fivefold serial dilutions of indicated strains on solid agar plates. Yeast strains isogenic to DF5, W303 and BY4741 were used for biochemical and genetic studies, and PJ69-7a for Y2H assays. All tagged strains and deletion mutants were constructed by a PCR-based strategy 32,33 and confirmed by western blot analysis and PCR, respectively. The $p r p 8^{*}$ allele ( $p r p 8(P 1384 L)$ ) was provided by G. Dittmar. For complementation studies, the complete $H U B 1$ and $S N U 66$ open reading frame (ORF), 1,000 bp of the upstream promoter and $500 \mathrm{bp}$ of its terminator were cloned into centromeric plasmids. For Hub1 overexpression, the HUB1 ORF was cloned into an integrative plasmid with the TEF2 promoter and $A D H 1$ terminator. To generate $5^{\prime}$-splice-site variants for the $S R C 1$ gene, the full-length ORF was cloned into a centromeric plasmid downstream of the GAL1-10 promoter. To generate the Hub1-Snu66 $\Delta \mathrm{N}$ linear fusion, DNA sequences encoding Hub1 plus a linker of nine glycine residues was inserted downstream of the $S N U 66$ promoter and upstream of the SNU66 ORF. The vectors $p E T 28 a-c$ or $p G E X-5 X 1$ were used for expression of $6 \times$ His-tagged or GST-tagged proteins, respectively, and $p G B D U C 1$ and $p G A D C 1$ for $\mathrm{Y} 2 \mathrm{H}$ assays. Plasmids with point mutations were constructed by site-directed mutagenesis using specific primers. Plasmodium falciparum genomic DNA (gift from K. Matuschewski) or a cDNA library of Arabidopsis thaliana (BioChain) were used as templates to generate the corresponding clones for interaction studies. For westernblot-based $S R C 1$ alternative splicing assays, a DNA sequence covering the $C U P 1-1$ promoter and TAP tag was integrated upstream of $S R C 1$ at its genomic loci. The CUP1-1 promoter was induced for $4 \mathrm{~h}$ in the presence of $200 \mu \mathrm{M} \mathrm{CuSO}_{4}$. For the construction of linear fusions of the splicing factors Snu66, Prp38 and Prp8 with Hub1 variants, DNA encoding $H U B 1$, hub1 $(D 22 A)$ and hub1-DD were cloned into vector pFA6a-natNT2. The C-terminal fusions were finally generated by a PCR-based strategy 32,33 . For the (GAL1-10promoter-driven) splicing reporter assays (Fig. $3 \mathrm{a}-\mathrm{c}$ ) and $S R C 1$ alternative splicing assays (Supplementary Fig. 13d), cells were grown to log phase in synthetic media containing 2\% lactate as carbon source ( $\mathrm{pH} 5.5$ ). Expression by the GAL1-10 promoter was induced for the indicated time by adding $2 \%$ galactose. The promoter was shut off by adding $2 \%$ glucose.

S. pombe strains are isogenic to JY741/746. $S$. pombe strains $\Delta h u b 1$ (YHY23P) and hub1-1 (YHY24P), and plasmids $p U R 19$ hub1+ and $p A L S K$ are gifts from H. Yashiroda ${ }^{8}$. Expression vectors for $S$. pombe studies were purchased from ATCC. For $S$. pombe $\Delta h u b 1$ complementation assays, $H U B 1$ cDNA was cloned into $p R E P 81$ (a vector containing thiamine-repressible $n m t 81$ promoter). For 5-FOA shuffle and complementation assays of $S$. pombe $\triangle$ snu66, the $S N U 66 \mathrm{ORF}$ with $1,000 \mathrm{bp}$ of the upstream promoter and $500 \mathrm{bp}$ of terminator were cloned into vectors $p U R 19$ (ura4+) or $p A L S K$ (leu2+). For complementation assays in $S$. pombe, competent cells were transformed with plasmids and incubated on selective media for 4 days at $30^{\circ} \mathrm{C}$. Fivefold serial dilutions were spotted on control and 5-FOA selection plates. The plates were incubated for 3-5 days at the temperatures indicated. Immunofluorescence in $S$. pombe was performed following a published protocol $^{34}$. 


\section{Antibodies}

The following antibodies were used: anti-haemagglutinin (HA, clone F-7) and anti-Clb2 (Santa Cruz Biotechnology); anti-Myc (clone 9E10), anti-Flag (clone M2) and anti-TAP (PAP) (all from Sigma-Aldrich); sheep anti-mouse Cy3-conjugated and HRP-coupled secondary antibodies (Jackson Immuno Research); antibodies against purified recombinant Hub1 (raised in rabbit); antibodies raised against two different Snu66 peptides (Eurogentec).

\section{Protein techniques and interaction studies}

Standard procedures were followed for purification of GST- and $6 \times$ His-tagged recombinant proteins from Escherichia coli. Proteins were dialysed and then cleaned on size exclusion columns. Because Snu66 tends to precipitate during dialysis, the salt concentration was increased to $300 \mathrm{mM} \mathrm{NaCl}$. Na-phosphate-based buffers were used for interaction studies, whereas HEPES-based buffer was used for proteins for structural studies. Isothermal titration calorimetry was performed to measure dissociation constants $\left(K_{\mathrm{d}}\right)$ using purified proteins and peptides. For Y2H assays, strain PJ69-7a was co-transformed with plasmids expressing the Gal4 DNA-binding domain fused to the baits, and the Gal4 activation domain fused to the preys. After 3 days of growth on selective media at $30^{\circ} \mathrm{C}$, fivefold dilutions of cultures were spotted on control plates and plates lacking the indicators histidine (-his) or adenine (-ade). The plates were further incubated for $2-5$ days at $30{ }^{\circ} \mathrm{C}$. For co-

immunoprecipitation assays, $100 \mathrm{OD}_{600}$ yeast cells were harvested at an $\mathrm{OD}_{600}=2.0$. Cells were washed once with PBS, and the pellet was frozen in liquid nitrogen. Cells were lysed either by bead beating (Retsch Instrument) or grinding in liquid nitrogen. The total yeast lysate prepared in a PBS-based buffer (1\% Triton X-100, protease inhibitors by Roche), was subjected to immunoprecipitation with bead-coupled antibodies for $3 \mathrm{~h}$ at $4{ }^{\circ} \mathrm{C}$. Beads were washed 4 times by rotating for $5 \mathrm{~min}$ at $4{ }^{\circ} \mathrm{C}$ in $1 \mathrm{ml}$ lysis buffer. Bound material was eluted by a dithiothreitol-containing hydroxyurea buffer ${ }^{32}$ followed by SDS-PAGE (4-16\%) and western blot analysis. For in vitro GST pull-down assays, $50 \mu \mathrm{g}$ of GST-fusion proteins bound to glutathione Sepharose beads were used to pull-down from equimolar amounts of purified proteins using the same buffer conditions as for co-immunoprecipitation experiments. Pull-down was performed for $1 \mathrm{~h}$ at $4{ }^{\circ} \mathrm{C}$; subsequent washing of beads, elution of bound material and electrophoresis was similar as for the co-immunoprecipitation assay. Protein bands were stained with PageBlue (Fermentas). For HIND pull-down assays, purified Snu66-N (wild type or RR-AA mutant) was covalently coupled to Sepharose beads (GE Healthcare). Pull-down of interacting proteins from yeast lysate was performed similar to the co-immunoprecipitation experiments. For immunoprecipitations using mammalian proteins, transiently transfected 293T cells were harvested, washed in ice-cold PBS and cell pellets were lysed at $4{ }^{\circ} \mathrm{C}$ for $30 \mathrm{~min}$ in 5 pellet volumes of lysis buffer $(50 \mathrm{mM}$ HEPES pH 7.2, $150 \mathrm{mM} \mathrm{NaCl}, 2 \mathrm{mM}$ EDTA, $1 \%$ Triton X-100, $1 \mathrm{mM}$ PMSF, and complete protease inhibitors (Roche)). After removal of cell debris by centrifugation $\left(10 \mathrm{~min}, 16,000 \mathrm{~g}, 4^{\circ} \mathrm{C}\right)$, lysates were incubated with anti-Flag-M2 affinity gel (Sigma-Aldrich). After $3 \mathrm{~h}$ the affinity matrix was washed 5 times in lysis buffer and eluted in Laemmli SDS buffer.

\section{Purification of Snu66-associated complexes}

For each purification (immunoprecipitation) reaction, $200 \mu \mathrm{g}$ anti-Snu66 antibody coupled to Dynal magnetic beads (Invitrogen) was used. Yeast lysate $(\sim 12 \mathrm{ml})$ was prepared from $3,500 \mathrm{OD}_{600}$ of yeast pellet by grinding with liquid nitrogen as described ${ }^{35}$.

Immunoprecipitation was performed at $4{ }^{\circ} \mathrm{C}$ for $3 \mathrm{~h}$. Beads were washed 4 times with $12 \mathrm{ml}$ lysis buffer containing $1 \%$ Triton $\mathrm{X}-100$ and rotating for $5 \mathrm{~min}$ at $4{ }^{\circ} \mathrm{C}$. Coimmunoprecipitation material was eluted by heating the beads at $65^{\circ} \mathrm{C}$ in the presence of $2 \%$ SDS then separated on 4-12\% SDS-PAGE. Protein bands were stained with PageBlue (Fermentas). Each sample lane was cut into nine gel slices, proteins were extracted, digested with trypsin, and analysed by Orbitrap mass spectrometry ${ }^{36}$. 


\section{Splicing-specific microarrays}

Splicing-specific microarrays, experimental design and data processing were described previously ${ }^{20,21}$.

\section{Northern blot analysis and RT-qPCR}

RNA was isolated for northern blot analysis using TRIzol (Invitrogen) and for RT-qPCR using an RNeasy kit (Quiagen). ${ }^{32} \mathrm{P}$-labelled HUB1 and SMT3 (SUMO)-specific probes were synthesized by random primer labelling (Ambion). Light cycler 480 was used for RTqPCR assays (Roche). Fifteen micrograms of total RNA was used for northern blot assay whereas $500 \mathrm{ng}$ of total RNA was used for reverse transcription.

\section{Splicing reporter assays}

The $\beta$-galactosidase assays were performed as described ${ }^{23}$. Site-directed mutagenesis was used to generate $5^{\prime}$-splice-site variants of the RP51-LacZ construct (gift from M. Rosbash). Survival on $\mathrm{CuSO}_{4}$-containing solid media for $A C T 1-C U P 1$ fusions was performed as described $^{24}$. Yeast strain yJU75, plasmids bearing PRP8 and prp8-101, and ACT1-CUP1 reporters wild type and $\mathrm{UuG}$ were gifts from C. Guthrie; plasmids prp8-R1753K, prp8-161 and prp8-162, and $A C T-C U P 1$ reporters A3C, BS-C and BS-G were gifts from M.

Konarska.

\section{Human cell lines, transfections and clones}

HEK $293 \mathrm{~T}$ and U2OS cells were maintained in DMEM (PAA) supplemented with 10\% FCS (Biochrom AG) at $37^{\circ} \mathrm{C}, 5 \% \mathrm{CO}_{2}$. HEK 293T cells were transfected using the calcium phosphate precipitation technique. Transfection of U2OS was performed using Lipofectamine 2000 (Invitrogen). The cDNA clone for Hs Snu66 (SART1) was purchased from Origene. The cDNA for Hs Hub1 (UBL5) was amplified by RT-PCR using total RNA from HeLa cells. Standard cloning techniques were used to generate expression constructs in pEGFP-N1 (Clontech) or $p 3 \times$ Flag-CMV-10 (Sigma-Aldrich) vectors.

\section{Immunofluorescence microscopy}

For immunofluorescence microscopy U2OS cells were seeded and transfected on glass coverslips (Roth). Cells were washed twice with PBS and fixed in 3.7\% fresh paraformaldehyde $\left(18 \mathrm{~min}\right.$, room temperature $\left.\left(24^{\circ} \mathrm{C}\right)\right)$. After incubation, paraformaldehyde was removed by aspiration and the cells were washed three times in PBS (5 min each). Permeabilization of cells was performed using PBS-Triton X-100 ( $0.4 \%$ for $6 \mathrm{~min})$, followed by three PBS-Tween (Tween 0.05\%; PBS-T) washing steps and blocking in PBS$\mathrm{T} / 2 \% \mathrm{BSA}$ for $1 \mathrm{~h}$ at room temperature. Coverslips were incubated with primary antibody for $2 \mathrm{~h}$ (dilution 1:200 in blocking buffer) and then washed three times in PBS-T (3 min each, room temperature). After incubation with secondary antibody (sheep anti-mouse Cy3conjugated, Jackson Immuno Research), cover slips were mounted using DAPI-containing mounting medium (Vectashield, Vector Labs). Images were acquired on a Zeiss AxioImager Z1 microscope equipped with an AxioCam MRm Rev.3 camera. Image acquisition was carried out using AxioVision Rel. 4.7 software (Zeiss).

\section{X-ray crystallography}

Complexes of recombinant $6 \times$ His-tagged Hub1 and HIND peptides were purified on S75 Superdex column. The protein buffer used for crystallization contained $20 \mathrm{mM}$ HEPES and $100 \mathrm{mM} \mathrm{NaCl}$ (pH 7.4). Crystallization of the complexes was carried out with the sittingdrop vapour diffusion method at $20^{\circ} \mathrm{C}$ by mixing equal volumes of protein complex and reservoir solution ( $0.2 \mathrm{M}$ ammonium iodide, $20 \%$ PEG 3350, pH 6.9). Crystals of both complexes appeared in several weeks and grew to a final size of $\sim 0.3 \times 0.2 \times 0.1 \mathrm{~mm}$. 
Crystals were plunged frozen after soaking for $\sim 30 \mathrm{~s}$ in a drop of a reservoir solution containing $30 \% \mathrm{v} / \mathrm{v}$ glycerol as a cryoprotectant. The crystals of the HIND-I-Hub1 complex belong to the space group $P 1$, with the unit cell $a=35.2 \AA, b=36.34 \AA, c=36.78 \AA$, $a=$ $83.44^{\circ}, \beta=89.85^{\circ}, \gamma=85.84^{\circ}$ and contained two complexes per an asymmetric unit. The HIND-II-Hub1 complex crystallized in space group $P 2_{1} 2_{1} 2$, with the unit cell dimensions $a$ $=36.72 \AA, \mathrm{b}=83.57 \AA, c=35.11 \AA$, and contained one complex per asymmetric unit.

The data sets, up to $1.4 \AA$ and $1.9 \AA$ at $90 \mathrm{~K}$, were collected on the MPG/GBF beamline BW6 at DESY, Hamburg, Germany using $1.05 \AA$ wavelength. The collected data were integrated, scaled and merged by XDS and XSCALE programs ${ }^{37}$. The structure was determined by molecular replacement using the Molrep program from the CCP4 suite ${ }^{38}$. The structure of ubiquitin, taken from the PDB entry 1UBI, was used as a probe structure. Models were then refined by Refmac5 (ref. 38) and rebuilt by XtalViev/Xfit ${ }^{39}$, followed by a subsequent Refmac5 refinement. Waters were added by Arp/warp ${ }^{40}$. The complete Hub1 molecule model had clear interpretable electron density, except for certain solvent exposed side chains, and those parts were omitted in the model. The structure of the Hub1-HIND-I complex has $94.9 \%$ of residues in the core, and $5.1 \%$ in allowed Ramachandran regions. For the Hub1-HIND-II complex those values are $93 \%$ and $7 \%$ respectively. Data collection and refinement statistics are summarized in Supplementary Table 2.

\section{NMR spectroscopy}

NMR spectroscopy was performed on a BRUKER AVANCE $600 \mathrm{MHz}$ spectrometer equipped with a Cryo-probe. For each sample a 1H-1D spectrum with a WATERGATE-5 water suppression was measured, and for $15 \mathrm{~N}$ labelled samples a fast HSQC spectrum ${ }^{41,42}$ was recorded. For the full-length $2 \mathrm{H}-15 \mathrm{~N}$ Snu66 experiment also a Trosy-HSQC was recorded $^{43,44}$.

\section{CD spectroscopy}

Synthetic peptides were solubilised in a buffer containing $20 \mathrm{mM} \mathrm{NaH}_{2} \mathrm{PO}_{4}, 100 \mathrm{mM} \mathrm{NaCl}$ (pH 7.6) at 0.01-0.02 $\mathrm{mM}$ concentration. CD spectra were obtained on a J-715 spectropolarimeter (Jasco J715 model). All spectra were recorded by using a quartz cell with a path length of $1 \mathrm{~mm}$. The parameters used for data acquisition were: response, $2 \mathrm{~s}$; scanning speed, $20 \mathrm{~nm} \mathrm{~min}{ }^{-1}$; bandwidth, $1.0 \mathrm{~nm}$; sensitivity, $5 \mathrm{mdeg}$; and step resolution, $0.1 \mathrm{~nm}$.

\section{Supplementary Material}

Refer to Web version on PubMed Central for supplementary material.

\section{Acknowledgments}

We thank U. Cramer and M. Kost for technical assistance, G. Dittmar, C. Guthrie, M. Konarska, K. Matuschewski, O. Nielsen, M. Rosbash and H. Yashiroda for materials, S. Uebel and C. Boulegue for mass spectrometric analysis and help, K. Hofmann for pointing out putative HIND elements in Prp38 proteins of plants, and M. Singh for initiating structural work. S.J. is supported by the Max Planck Society, Deutsche Forschungsgemeinschaft, Fonds der chemischen Industrie, Center for Integrated Protein Science Munich and RUBICON EU Network of Excellence; T.A.H. by the Max Planck Society; M.A. by NIH (GM040478).

\section{References}

1. Hochstrasser M. Origin and function of ubiquitin-like proteins. Nature. 2009; 458:422-429. [PubMed: 19325621]

2. McNally T, et al. Structural analysis of UBL5, a novel ubiquitin-like modifier. Protein Sci. 2003; 12:1562-1566. [PubMed: 12824502] 
3. Ramelot TA, et al. Solution structure of the yeast ubiquitin-like modifier protein Hub1. J. Struct. Funct. Genomics. 2003; 4:25-30. [PubMed: 12943364]

4. Wilkinson CR, et al. Ubiquitin-like protein Hub1 is required for pre-mRNA splicing and localization of an essential splicing factor in fission yeast. Curr. Biol. 2004; 14:2283-2288. [PubMed: 15620657]

5. Friedman JS, Koop BF, Raymond V, Walter MA. Isolation of a ubiquitin-like (UBL5) gene from a screen identifying highly expressed and conserved iris genes. Genomics. 2001; 71:252-255. [PubMed: 11161819]

6. Dittmar GA, Wilkinson CR, Jedrzejewski PT, Finley D. Role of a ubiquitin-like modification in polarized morphogenesis. Science. 2002; 295:2442-2446. [PubMed: 11923536]

7. Lüders J, Pyrowolakis G, Jentsch S. The ubiquitin-like protein HUB1 forms SDS-resistant complexes with cellular proteins in the absence of ATP. EMBO Rep. 2003; 4:1169-1174. [PubMed: 14608371]

8. Yashiroda H, Tanaka K. Hub1 is an essential ubiquitin-like protein without functioning as a typical modifier in fission yeast. Genes Cells. 2004; 9:1189-1197. [PubMed: 15569151]

9. Benedetti C, Haynes CM, Yang Y, Harding HP, Ron D. Ubiquitin-like protein 5 positively regulates chaperone gene expression in the mitochondrial unfolded protein response. Genetics. 2006; 174:229-239. [PubMed: 16816413]

10. Hazbun TR, et al. Assigning function to yeast proteins by integration of technologies. Mol. Cell. 2003; 12:1353-1365. [PubMed: 14690591]

11. Wahl MC, Will CL, Luhrmann R. The spliceosome: design principles of a dynamic RNP machine. Cell. 2009; 136:701-718. [PubMed: 19239890]

12. Ramage R, et al. Synthetic, structural and biological studies of the ubiquitin system: the total chemical synthesis of ubiquitin. Biochem. J. 1994; 299:151-158. [PubMed: 8166633]

13. Bayer P, et al. Structure determination of the small ubiquitin-related modifier SUMO-1. J. Mol. Biol. 1998; 280:275-286. [PubMed: 9654451]

14. Dikic I, Wakatsuki S, Walters KJ. Ubiquitin-binding domains-from structures to functions. Nature Rev. Mol. Cell Biol. 2009; 10:659-671. [PubMed: 19773779]

15. Song J, Zhang Z, Hu W, Chen Y. Small ubiquitin-like modifier (SUMO) recognition of a SUMO binding motif: a reversal of the bound orientation. J. Biol. Chem. 2005; 280:40122-40129. [PubMed: 16204249]

16. Makarova OV, Makarov EM, Luhrmann R. The 65 and 110 kDa SR-related proteins of the U4/ U6.U5 tri-snRNP are essential for the assembly of mature spliceosomes. EMBO J. 2001; 20:25532563. [PubMed: 11350945]

17. Stevens SW, Abelson J. Purification of the yeast U4/U6.U5 small nuclear ribonucleoprotein particle and identification of its proteins. Proc. Natl Acad. Sci. USA. 1999; 96:7226-7231. [PubMed: 10377396]

18. Stevens SW, et al. Composition and functional characterization of the yeast spliceosomal pentasnRNP. Mol. Cell. 2002; 9:31-44. [PubMed: 11804584]

19. Fabrizio $P$, et al. The evolutionarily conserved core design of the catalytic activation step of the yeast spliceosome. Mol. Cell. 2009; 36:593-608. [PubMed: 19941820]

20. Clark TA, Sugnet CW, Ares M Jr. Genomewide analysis of mRNA processing in yeast using splicing-specific microarrays. Science. 2002; 296:907-910. [PubMed: 11988574]

21. Burckin T, et al. Exploring functional relationships between components of the gene expression machinery. Nature Struct. Mol. Biol. 2005; 12:175-182. [PubMed: 15702072]

22. Keren H, Lev-Maor G, Ast G. Alternative splicing and evolution: diversification, exon definition and function. Nature Rev. Genet. 2010; 11:345-355. [PubMed: 20376054]

23. Jacquier A, Rodriguez JR, Rosbash M. A quantitative analysis of the effects of $5^{\prime}$ junction and TACTAAC box mutants and mutant combinations on yeast mRNA splicing. Cell. 1985; 43:423430. [PubMed: 3935321]

24. Lesser CF, Guthrie C. Mutational analysis of pre-mRNA splicing in Saccharomycescerevisiae using a sensitive new reporter gene,CUP1. Genetics. 1993; 133:851-863. [PubMed: 8462846] 
25. Chen M, Manley JL. Mechanisms of alternative splicing regulation: insights from molecular and genomics approaches. Nature Rev. Mol. Cell Biol. 2009; 10:741-754. [PubMed: 19773805]

26. Davis CA, Grate L, Spingola M, Ares M. Test of intron predictions reveals novel splice sites, alternatively spliced mRNAs and new introns in meiotically regulated genes of yeast. Nucleic Acids Res. 2000; 28:1700-1706. [PubMed: 10734188]

27. Rodríguez-Navarro S, Igual JC, Pérez-Ortín JE. SRC1: an intron-containing yeast gene involved in sister chromatid segregation. Yeast. 2002; 19:43-54. [PubMed: 11754482]

28. Grund SE, et al. The inner nuclear membrane protein Src1 associates with subtelomeric genes and alters their regulated gene expression. J. Cell Biol. 2008; 182:897-910. [PubMed: 18762579]

29. Kuhn AN, Kaufer NF. Pre-mRNA splicing in Schizosaccharomyces pombe: regulatory role of a kinase conserved from fission yeast to mammals. Curr. Genet. 2003; 42:241-251. [PubMed: 12589463]

30. Okazaki K, Niwa O. mRNAs encoding zinc finger protein isoforms are expressed by alternative splicing of an in-frame intron in fission yeast. DNA Res. 2000; 7:27-30. [PubMed: 10718196]

31. Kress TL, Krogan NJ, Guthrie C. A single SR-like protein, Npl3, promotes pre-mRNA splicing in budding yeast. Mol. Cell. 2008; 32:727-734. [PubMed: 19061647]

32. Knop M, et al. Epitope tagging of yeast genes using a PCR-based strategy: more tags and improved practical routines. Yeast. 1999; 15:963-972. [PubMed: 10407276]

33. Janke C, et al. A versatile toolbox for PCR-based tagging of yeast genes: new fluorescent proteins, more markers and promoter substitution cassettes. Yeast. 2004; 21:947-962. [PubMed: 15334558]

34. Hagan, IM.; Ayscough, KR. Protein Localization by Fluorescence Microscopy: A Practical Approach. Allan, VJ., editor. Oxford Univ. Press; 2000. p. 179-205.

35. Ansari A, Schwer B. SLU7 and a novel activity, SSF1, act during the PRP16-dependent step of yeast pre-mRNA splicing. EMBO J. 1995; 14:4001-4009. [PubMed: 7664739]

36. Steen H, Mann M. The abc's (and xyz's) of peptide sequencing. Nature Rev. Mol. Cell Biol. 2004; 5:699-711. [PubMed: 15340378]

37. Kabsch W. Automatic processing of rotation diffraction data from crystals of initially unknown symmetry and cell constants. J. Appl. Cryst. 1993; 26:795-800.

38. Collaborative Computational Project 4. The CCP4 suite: programs for protein crystallography. Acta Crystallogr. D. 1994; 50:760-763. [PubMed: 15299374]

39. McRee DE. XtalView/Xfit—a versatile program for manipulating atomic coordinates and electron density. J. Struct. Biol. 1999; 125:156-165. [PubMed: 10222271]

40. Lamzin VS. Automated refinement of protein models. Acta Crystallogr. D. 1993; 49:129-147. [PubMed: 15299554]

41. Liu M, et al. Improved WATERGATE pulse sequences for solvent suppresion in NMR spectroscopy. J. Magn. Reson. 1998; 132:125-129.

42. Mori S, Abeygunawardana C, Johnson MO, van Zijl PC. Improved sensitivity of HSQC spectra of exchanging protons at short interscan delays using a new fast HSQC (FHSQC) detection scheme that avoids water saturation. J. Magn. Reson. B. 1995; 108:94-98. [PubMed: 7627436]

43. Zhu G, Kong XM, Sze KH. Gradient and sensitivity enhancement of 2D TROSY with water flipback, 3D NOESY-TROSY and TOCSY-TROSY experiments. J. Biomol. NMR. 1999; 13:77-81. [PubMed: 21080266]

44. Pervushin KV, Wider G, Wüthrich K. Single transition-to-single transition polarization transfer (ST2-PT) in [15N,1H]-TROSY. J. Biomol. NMR. 1998; 12:345-348. [PubMed: 21136330] 

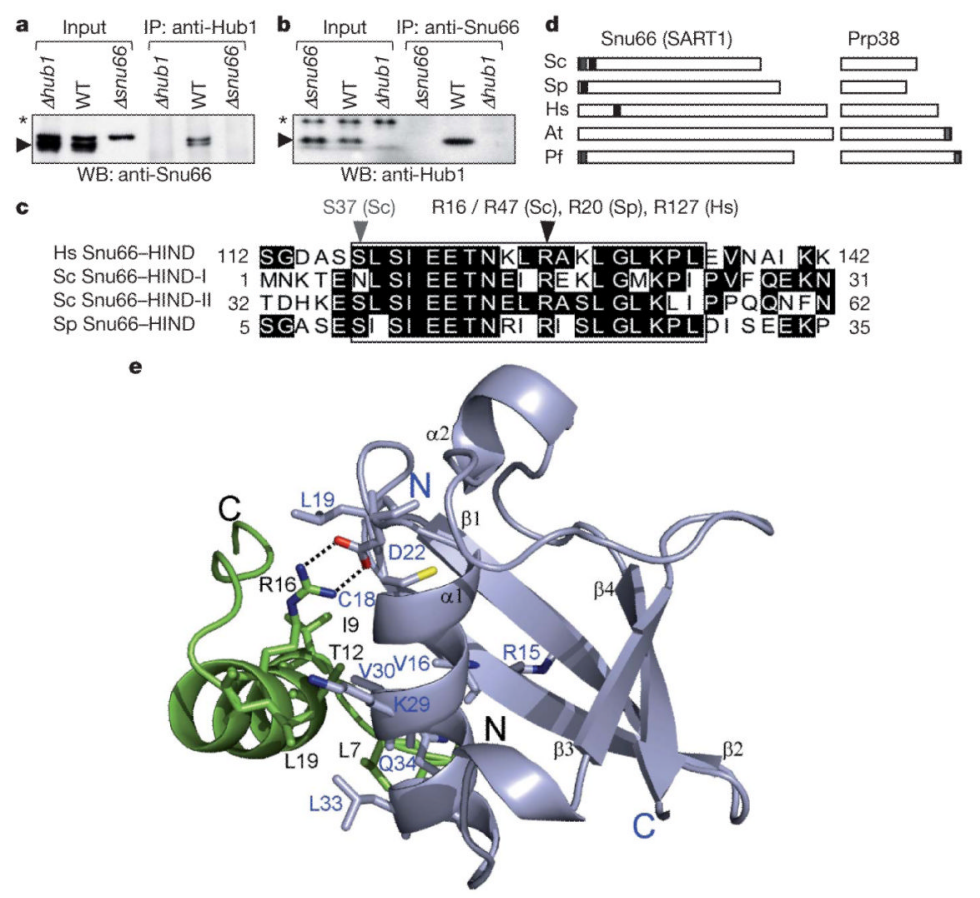

Figure 1. Hub1 binds to HIND

a, Immunoprecipitation (IP) of Hub1 and co-immunoprecipitation of Snu66 from the indicated yeast strains. WB, western blot; WT, wild type. b, Immunoprecipitation of Snu66 and co-immunoprecipitation of Hub1 from the indicated yeast strains (asterisks denote cross-reacting signals). c, Clustal-W alignment of Snu66-HINDs from different organisms. Numbers give the positions of amino acids, and arrows indicate conserved Arg and Ser residues. At, Arabidopsis thaliana; Hs, Homo sapiens; Pf, Plasmodium falciparum; Sc, Saccharomyces cerevisiae; Sp, Schizosaccharomyces pombe. d, Diagram of homologues of the spliceosomal proteins Snu66 (SART1) and Prp38, and the occurrence of HINDs (shaded). e, Crystal structure of Hub1 in complex with HIND-I peptide shown as ribbon plot. The interaction interface is formed by a salt bridge between Arg 16(47) (numbers in parentheses refer to HIND-II) of Snu66 and Asp 22 of Hub1, accompanied by a patch of hydrophobic interactions. The hydrophobic residues of HINDs that participate in the Hub1binding interface are Leu 7(38), Ile 9(40), Thr 12(43), Ile 15 (Leu 46), Arg 16(47) (C $\beta$ and C $\gamma$ ), Leu 19(50), Met 21 (Leu 52) and Ile 24(55). On Hub1, the interface is formed by Leu 19, Val 30, and additionally Met 1, Val 16, Lys 17 (C $\beta, C \gamma, C \Delta)$, Cys 18, Lys 29 (C $\beta, C \gamma$, $\mathrm{C} \Delta$ ) and Leu 33. 

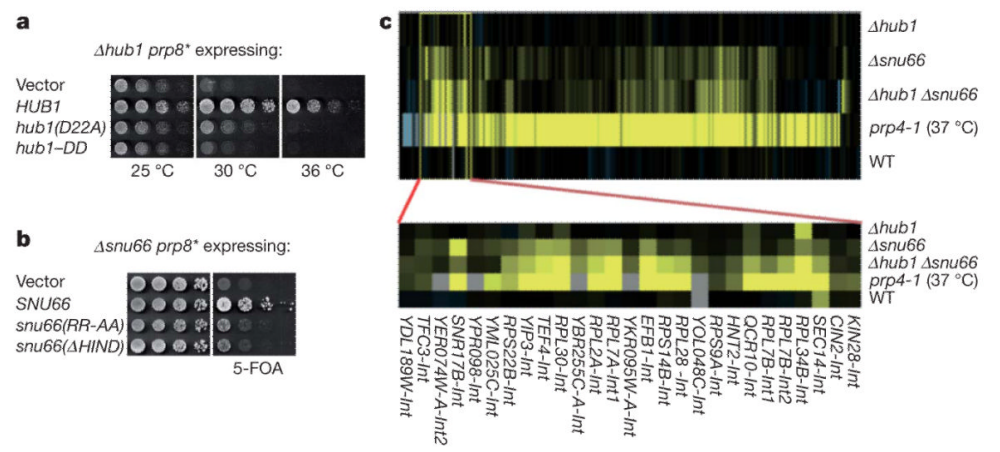

Figure 2. Functional links to splicing

a, Rescue of synthetic sickness of $\triangle h u b 1$ prp $8^{*}$ by expression of $H U B 1$ wild type, but not $h u b 1(D 22 A)$ and $h u b 1-D D$. b, Rescue of synthetic lethality of $\Delta s n u 66$ prp $8^{*}$ by expression of $S N U 66$ wild type, but not by $\operatorname{snu} 66(R R-A A)$ or $\operatorname{snu66}(\Delta H I N D)$. Wild-type $S N U 66$ expressed from a $U R A 3$-containing plasmid was shuffled out by counter-selection on 5fluoroorotic acid (5-FOA) plates. c, Microarray analysis for splicing defects. Diagram of intron accumulation (yellow) in strains deleted of HUB1 and SNU66 (data in Supplementary Table 3). The top panel shows relative splicing efficiency for almost all yeast introns as measured by splicing sensitive microarrays ${ }^{20,21}$. In each case RNA is derived from the indicated mutant, and wild-type RNA (comparing two different RNA preparations from wild-type cells) is used for reference. The temperature-sensitive splicing mutant prp4-1 is the positive control. The bottom panel is an expanded view of a set of transcripts that are particularly affected in $\triangle h u b 1 \triangle$ snu 66 and also includes an intron of $R P L 34 B$ that is mildly affected in $\Delta h u b 1$. 

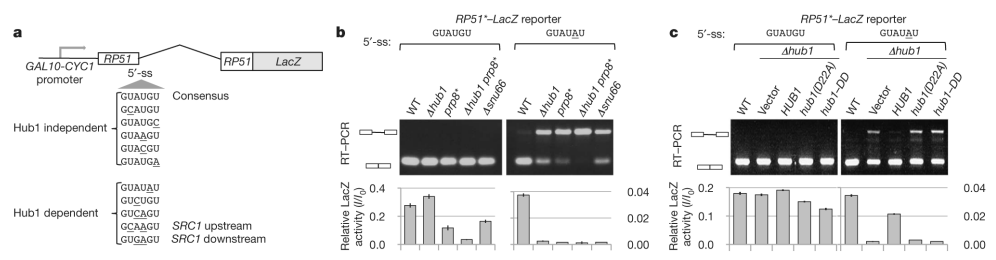

Figure 3. Splice-site usage

a, Scheme for the requirement of Hub1 for efficient utilization of canonical and noncanonical $5^{\prime}$ splice sites ( $5^{\prime}$-ss; underlines mark changes) inserted into RP51*-LacZ fusions $^{23}$. The individual $5^{\prime}$ splice sites of $S R C 1$ in the context of the reporter assay were used. b, c, Splicing defects in wild type and mutants with canonical (GUAUGU) and noncanonical (GUAUAUU) 5' splice sites. RT-PCR assay of total RNA isolated from yeast expressing different version of intron-containing $R P 51^{*}-L a c Z$ fusions. Bottom panels show ratios of corresponding LacZ ( $\beta$-galactosidase) activities $(I)$ to the activity from an intronless $\left(I_{0}\right)$ construct. Error bars show standard deviation of three samples. Strains in $\mathbf{c}$ are $\triangle h u b 1$ and complemented by $H U B 1$ alleles. 


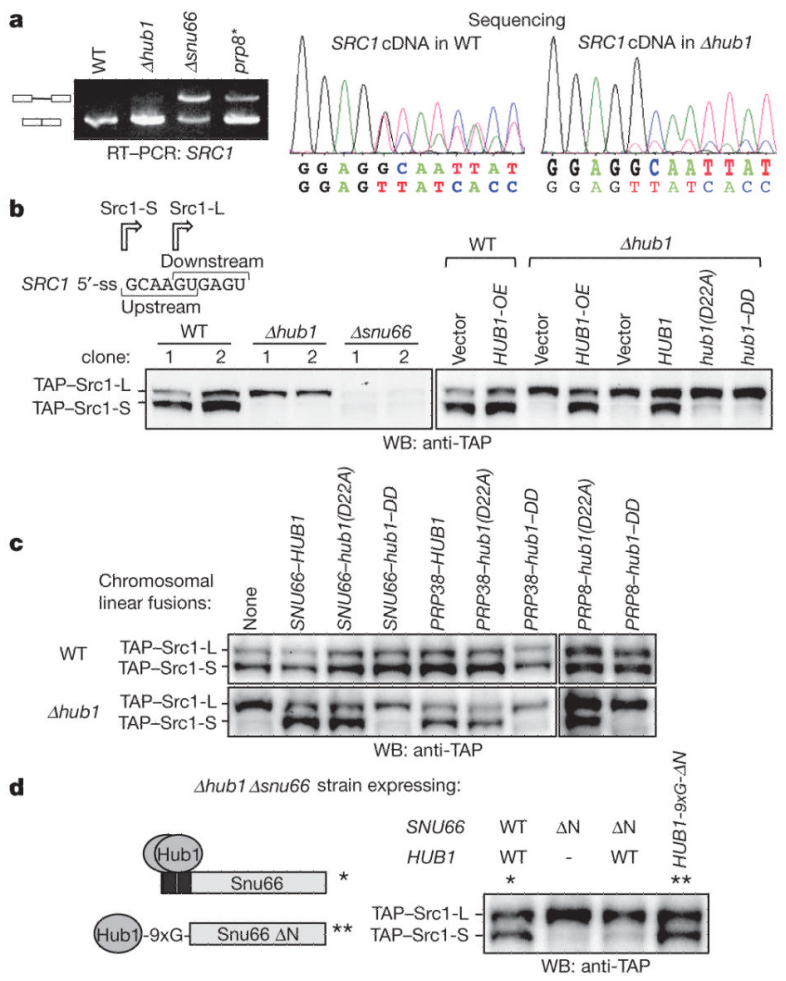

Figure 4. Alternative splicing of $S R C 1$

a, RT-PCR assay of SRC1 transcripts in different mutants (left panel), and sequencing of $S R C 1$ cDNA from wild-type (WT) and $\triangle h u b 1$ strains (right panels). Only general splicing defects of $\Delta s n u 66$ and $p r p 8^{*}$ mutants are detectable by RT-PCR as the $5^{\prime}$ splice sites of $S R C 1$ are overlapping, but alternative splicing defects via the upstream $5^{\prime}$ splice site in the $\triangle h u b 1$ strain are revealed by sequencing. b. Chromosomal $S R C 1$ was N-terminally TAP tagged to detect the two gene products (TAP-Src1-L, TAP-Src1-S) generated by alternative splicing. Splicing via the upstream (GCAAAGU) and downstream (GUGAGU) $5^{\prime}$ splice sites $\left(5^{\prime}\right.$-ss) is indicated (top left). Alternative splicing defects (usage of the upstream $5^{\prime}$ splice site) in $\Delta h u b 1$ and general splicing defects in $\triangle$ snu 66 were detected by western blotting using TAP-tag-specific antibodies. Wild-type and mutant strains, and $\triangle h u b 1$ strains expressing Hub1 variants, or which overexpress $H U B 1$, were used. c, $S R C 1$ alternative splicing is restored in $\triangle$ hub1 harbouring chromosomally expressed linear fusions of PRP8, $P R P 38$ and $S N U 66$ genes with $H U B 1$ or hub1(D22A) but not with hub1-DD (assay similar to b). d, $S R C 1$ alternative splicing is supported by a linear fusion of Hub1 with Snu66 $\triangle \mathrm{N}$ in a $\Delta$ hub1 $\Delta$ snu66 strain. The $\Delta h u b 1 \Delta s n u 66$ strain with TAP-tagged Src1 was transformed with plasmids expressing $H U B 1, S N U 66$, or a construct encoding Hub1 fused to the N terminus of the Snu66 variant that lacks the HIND region $(\Delta N)$, separated by a poly-glycine $(9 \times \mathrm{G})$ linker. Asterisks indicate the two ways of Hub1-Snu66 complex formation. 